\title{
Capacity gain and design trade-offs for partial-duplex OFDM wireless communications
}

\author{
Hamid R. Barzegar ${ }^{1}$, Luca Reggiani ${ }^{1}$ and Laura Dossi2 ${ }^{*}$ (i)
}

\begin{abstract}
A hybrid transmission method between classical half-duplex and full-duplex is here considered for point-to-point orthogonal frequency division multiplexing (OFDM) links experiencing frequency selective fading. This partial-duplex solution uses only a portion of the available bandwidth for full-duplex transmission. It aims to increase the overall bidirectional system rate w.r.t. an equivalent half-duplex system, relaxing at the same time the high self-interference cancelation requirements that practical full-duplex systems have to provide. In this paper, we analyze the regions of self-interference cancelation values where partial-duplex systems outperform half-duplex ones in terms of achievable spectral efficiency. We investigate the potential of the proposed hybrid method by deriving the analytical distributions of the spectral efficiency gain regions in the presence of frequency selective Rayleigh fading. For different strategies in the selection of the sub-carriers that operate in full-duplex, we highlight the role of the different parameters involved and the peculiarity of this system in terms of design flexibility.
\end{abstract}

\section{Introduction}

The interest for wireless full-duplex (FD) communication in the future $5 \mathrm{G}$ and beyond networks is increasing due to its potential of contributing to some of the ambitious goals in the evolution of the next generation of wireless communication: increased distance and capacity of the wireless links, spectrum virtualization, and enhanced interference coordination [1, 2]. However, one of the most important deterrents in implementing an FD wireless system is the impact of the self-interference signal that is generated locally by the transmission device. The amplitude of this echo, in fact, is tens of decibels above the received signal of interest, which comes from the other transmitter and is highly attenuated by the channel propagation path loss and fading. For this reason, the self-interference cancelation (SIC) capability is the crucial technology that can make possible the FD promising throughput improvements $[3,4]$.

One of the earliest reports on experimental demonstrations of FD communication for narrow-band wireless communication systems was presented in 1998 [5]. Since that time, many researchers have investigated this topic

*Correspondence: laura.dossi@ieiit.cnr.it

${ }^{2}$ IEIIT, CNR, Milan, Italy

Full list of author information is available at the end of the article and proposed different methods to extend the implementation to larger bandwidths and have studied solutions for the transmission of a signal in FD, based on several types of SIC schemes, from the analog radio frequency domain to the digital baseband domain. One of the simplest SIC methods consists of exploiting transmit and receive directional antennas to decouple the transmitted from the received signals [6]; other solutions are also based on antenna cancelation methods [7], relying on proper antenna positions, beam-forming tuning, or multiple-input multiple-output (MIMO).

In [8-11], the innovative approach is in the antenna design, supporting multiple antennas two-way transmission with multiple levels of cancelation of the selfinterference signal. On the other hand, [12] presents the design and implementation of a single antenna with high values of SIC till $110 \mathrm{~dB}$ measured for Wi-Fi signals in dense indoor office environments.

In [13], an auxiliary transmit path at radio frequency is used to feed a cancelation signal, which is a pre-processed version of the transmit signal, in order to match exactly the self-interference signal and cancel it.

In [14], a prototype of the FD design is realized on a software-defined radio platform; this design combines a dual-polarization antenna-based analog part with a digital self-interference canceler that operates in real time. The 
results from tests on this prototype confirm that the proposed FD system achieves about 1.9 times higher throughput than the corresponding half-duplex (HD) system, even if for short distances.

In [15], the maximum achievable FD link range is reported as $30 \mathrm{~m}$. The short-range capability of FD systems, reported in many studies, is another way of expressing the strong impact of the residual self-interference on the system performance.

In [16], we proposed the partial-duplex (PD) system, a hybrid solution where HD and FD coexist on the same link and at the same time, differently from other studies.

In [17], the hybrid solution named $X$-duplex switches between HD and FD transmission, depending on the level of the self-interference. The authors in [18-20] evaluate the performance of FD wireless communication networks consisting of base stations with FD capability and legacy HD users.

In [21-23], the hybrid solution named $\alpha$-duplex is designed for bidirectional single-carrier transmission where the bands for the two directions are contiguous. The proposed solution is to overlap partially the two bands (the parameter $\alpha$ is the percentage of overlapped bandwidth), enlarging the bandwidth in each direction and increasing the capacity of each directional link. This objective is achieved at the expense of losing the signal orthogonality in the spectral domain. The authors report a performance gain on the bidirectional link through a proper selection of the pulse shaping and the related matched filtering in order to optimize the signal separation at the receiver.

On the contrary, our PD hybrid solution is a bidirectional communication link which operates in FD mode only in a portion of the bandwidth, partitioning the overall bandwidth into one FD segment serving simultaneously both directions and two HD segments, one for each direction.

The PD system investigated here is based on multicarrier transmission on frequency selective channels as in [16], where different criteria can be adopted to partition the bandwidth in FD and HD segments. Two different strategies in the assignment of the FD sub-carriers are revised in Section 4.1, one based on a block allocation, named block partial-duplex, and the other one based on selective allocation, named selective partial-duplex. The PD scheme has obviously two extreme cases, the full $\mathrm{HD}$ and the full FD, and can be applied either to pointto-point high-capacity links or to point-to-multi-point links in cellular scenarios. W.r.t. FD, our approach balances a capacity loss, coming from the partial use of the bandwidth simultaneously in the two transmission directions, with a decrease of the SIC requirements. Here, we limit our study on symmetrical point-to-point links where the transceiver equipments at both ends have equivalent characteristics, e.g., noise figure and SIC capability, and the propagation introduces path loss and Rayleigh frequency selective fading. The numerical results are then extended to a standardized International Telecommunication Union - vehicular A (ITU-VehA) frequency selective fading model.

The novel contribution of the paper is the definition and the derivation of the theoretical limits of a PD approach in terms of the achievable spectral efficiency gains w.r.t. a classical HD system and the investigation of the role of the main system parameters involved in the PD design.

We remark that a PD approach is intended to be complementary to the implementation of an efficient selfinterference canceler; the main motivation for the adoption of a PD scheme is the search of encoding solutions that take advantage from the bandwidth portion with higher SNR, possibly exploited in FD mode, for relaxing the high performance targets of the SIC scheme. In order to reveal the potential advantage of the method, the analytical results assume an ideal receiver model, neglecting possible non-idealities as imperfect synchronization or amplifier nonlinearities or possible leakages from FD to HD sub-carriers due to impairments present in a real radio frequency receiver. On the other hand, these impairments have been taken into account in the simulation results of the channel model in order to show their impact on the SIC margin w.r.t. that predicted by the analysis.

In the sequel, Section 2 revises the main concepts of the PD wireless solution. The system model and the strategies adopted for PD are introduced in Section 3. Then, in Section 4, we present the analysis of the capacity gains and of the related SIC design trade-offs in AWGN and frequency selective channels. Finally, in Section 5, the numerical results, obtained analytically and by the simulations, are reported and discussed.

\section{Partial-duplex}

PD is a hybrid solution between classical HD and FD. Its rationale is the increase of the overall bidirectional system data rate w.r.t. HD operation, with a contemporary relaxation of the high SIC requirements. In the proposed PD approach, the PD parameter (PDP) is the fundamental factor that returns the fraction of the overall spectrum that will operate in FD mode. We will show that, in the presence of frequency selective fading, a smart choice of the portion of the bandwidth to be used in FD can maximize the spectral efficiency while operating with a self-interference canceler of lower performance and complexity w.r.t. that required in an FD system.

Our reference application is a multi-carrier transmission where, given a value of PDP, the $N$ sub-carriers are 
partitioned into two groups $\Pi_{\mathrm{HD}}$ and $\Pi_{\mathrm{FD}}$, consisting of $N_{\mathrm{HD}}$ and $N_{\mathrm{FD}}$ sub-carriers, that operate on one and two directions, respectively (considering two generic nodes $\mathrm{A}$ and $\mathrm{B}$, the two directions are $\mathrm{A}$ to $\mathrm{B}$ and $\mathrm{B}$ to $\mathrm{A}$ ), i.e.:

$$
\begin{aligned}
& N_{\mathrm{FD}}=\mathrm{PDP} \cdot N, \\
& N_{\mathrm{HD}}=N-N_{\mathrm{FD}}=(1-\mathrm{PDP}) \cdot N .
\end{aligned}
$$

We assume that $N_{\mathrm{FD}}=\mathrm{PDP} \cdot N$ is approximated to the closest integer value and $N_{\mathrm{HD}}$ to the closest even number, so providing the same capacity on the two link directions.

In order to analyze the performance of PD systems, we derive the spectral efficiency gain region as the region of SIC capability in which PD system outperforms HD in terms of spectral efficiency. Our approach is similar to the approach adopted in [24, 25]. In [24], the authors define the rate gain region on the received signal-of-interest strength, while here we define it on the capability of SIC. The spectral efficiency region is obtained by the condition $\eta_{\mathrm{PD}}>\eta_{\mathrm{HD}}$, where $\eta_{\mathrm{PD}}$ and $\eta_{\mathrm{HD}}$ are the PD and HD spectral efficiencies, respectively.

In a multi-carrier system with $N$ sub-carriers, a sum spectral efficiency region will be investigated according to the spectral efficiency $\eta$ [bits/sub-carrier] averaged on the overall bandwidth as:

$$
\eta=\frac{1}{N} \sum_{i=1}^{N} \eta_{i}
$$

where $\eta_{i}$ are the spectral efficiencies provided on each sub-carrier $(i=1,2, \cdots, N)$. Considering that each subcarrier can work in HD, i.e., serving only one direction of the link, or in FD, serving both directions, the spectral efficiency formula for $\eta_{i}$ on the $i$ th sub-carrier varies depending whether it is operated in HD or FD mode, respectively as $\eta_{\mathrm{HD}, i}$ and $\eta_{\mathrm{FD}, i}$. Such spectral efficiency values depend on the signal-to-noise ratio or signal-tointerference-plus-noise values, namely $\gamma_{\mathrm{HD}, i}$ and $\gamma_{\mathrm{FD}, i}$ on each sub-carrier, as:

$$
\begin{aligned}
& \eta_{H \mathrm{D}, i}=\log _{2}\left(1+\gamma_{\mathrm{HD}, i}\right), \\
& \eta_{\mathrm{FD}, i}=2 \cdot \log _{2}\left(1+\gamma_{\mathrm{FD}, i}\right),
\end{aligned}
$$

where the derivation computation of $\gamma_{\mathrm{HD}, i}$ and $\gamma_{\mathrm{FD}, i}$ will be discussed in Section 4. Therefore, the Shannon spectral efficiency (1) in the PD case is specified as:

$$
\eta_{\mathrm{PD}}=\frac{1}{N}\left(\sum_{i \in \Pi_{\mathrm{FD}}} \eta_{\mathrm{FD}, i}+\sum_{i \in \Pi_{\mathrm{HD}}} \eta_{\mathrm{HD}, i}\right)
$$

and the PD spectral efficiency gain $\eta_{\mathrm{G}}[\%]$ is defined w.r.t. the HD spectral efficiency $\eta_{\mathrm{HD}}=\frac{1}{N} \sum_{i=1}^{N} \eta_{\mathrm{HD}, i}$ as:

$$
\eta_{\mathrm{G}}[\%]=\frac{\eta_{\mathrm{PD}}-\eta_{\mathrm{HD}}}{\eta_{\mathrm{HD}}} 100 .
$$

On the contrary, in the presence of random frequency selective fading, for each $j$ th fading realization, the generic $i$ th sub-carrier experiences the spectral efficiencies $\eta_{\mathrm{FD}, i, j}$ or $\eta_{\mathrm{HD}, i, j}$ respectively in FD and HD, and the $j$ th sum spectral efficiencies $\eta_{\mathrm{PD}, j}$ and $\eta_{\mathrm{HD}, j}$ are evaluated by:

$$
\begin{aligned}
& \eta_{\mathrm{PD}, j}=\frac{1}{N}\left(\sum_{i \in \Pi_{\mathrm{FD}}} \eta_{\mathrm{FD}, i, j}+\sum_{i \in \Pi_{\mathrm{HD}}} \eta_{\mathrm{HD}, i, j}\right), \\
& \eta_{\mathrm{HD}, j}=\frac{1}{N}\left(\sum_{i=1}^{N} \eta_{\mathrm{HD}, i, j}\right) .
\end{aligned}
$$

Therefore, given the statistical fluctuations of $\eta_{\mathrm{FD}, i, j}$ and $\eta_{\mathrm{HD}, i, j}$ per sub-carrier and per fading realization, two definitions of the spectral efficiency gain regions for the PD scheme are considered:

- The former considers the average values of the spectral efficiencies $E_{j}\left[\eta_{\mathrm{PD}, j}\right]$ and $E_{j}\left[\eta_{\mathrm{HD}, j}\right]$. The efficiency gain region (4) is defined by:

$$
\begin{aligned}
& \sum_{i \in \Pi_{\mathrm{FD}}} E_{j}\left[\eta_{\mathrm{FD}, i, j}\right]+\sum_{i \in \Pi_{\mathrm{HD}}} E_{j}\left[\eta_{\mathrm{HD}, i, j}\right] \geq \\
& \sum_{i \in\left\{\Pi_{\mathrm{FD}} \cup \Pi_{\mathrm{HD}}\right\}} E_{j}\left[\eta_{\mathrm{HD}, i, j}\right],
\end{aligned}
$$

which is simplified to:

$$
\sum_{i \in \Pi_{\mathrm{FD}}} E_{j}\left[\eta_{\mathrm{FD}, i, j}\right] \geq \sum_{i \in \Pi_{\mathrm{FD}}} E_{j}\left[\eta_{\mathrm{HD}, i, j}\right]
$$

and it is satisfied if:

$$
E_{j}\left[\eta_{\mathrm{FD}, i, j}\right] \geq E_{j}\left[\eta_{\mathrm{HD}, i, j}\right], \forall i \in \Pi_{\mathrm{FD}} .
$$

- The latter imposes a limit on the occurrence of the event $\eta_{\mathrm{PD}, j}<\eta_{\mathrm{HD}, j}$. Given the condition:

$$
P_{\text {out }}=\operatorname{Prob}_{j}\left[\eta_{\mathrm{PD}, j}<\eta_{\mathrm{HD}, j}\right]=0.01,
$$

or, equivalently, $\operatorname{Prob}_{j}\left[\eta_{\mathrm{PD}, j} \geq \eta_{\mathrm{HD}, j}\right]=0.99$, from (6) and (7), the gain region is defined as:

$$
\operatorname{Prob}_{j}\left(\eta_{\mathrm{FD}, i, j}>\eta_{\mathrm{HD}, i, j}\right) \geq 0.99, \forall i \in \Pi_{\mathrm{FD}} .
$$

This definition corresponds to a more restrictive efficiency gain region than the previous one.

In multi-carrier systems with frequency selective fading, different strategies of allocation of HD and FD portions can be considered for taking advantage of the system flexibility. Two schemes have been already proposed in [16]: a selective strategy (selective partial-duplex (SPD)) and a block strategy (block partial-duplex (BPD)). In 
BPD, the sub-carriers selected for FD mode are adjacent, as in a single block. Without any selection strategy on the block, we can assume to choose it centered in the available spectrum; this is the allocation strategy that reproduces the method proposed in [21-23] in order to increase the throughput in single-carrier systems, by partially overlapping of the spectrum of the two adjacent bandwidths assigned to the two directions of the link. On the other hand, the SPD strategy exploits the maximum available selectivity of multi-carrier transmission, allowing the selection of the FD sub-carriers as those ones with the higher signal-to-interference-plusnoise ratio $\gamma_{\mathrm{FD}, i, j}$ thus providing potentially the best spectral efficiency gain. In [16], the numerical results have already highlighted the advantage from a flexible design of PD systems in the combination of SIC and PDP parameters, with the aim of increasing spectral efficiency performance for a fixed link distance or, alternatively, improving link distance performance for a target spectral efficiency. Depending on the characteristics of the random fading and on the PD strategy, numerical results in [16] showed indeed how longer distances can be reached for reasonable SIC capabilities by decreasing PDP w.r.t. the high demanding SIC capability in a full FD system.

\section{System model}

Let us consider a bidirectional link between the two nodes, $\mathrm{A}$ and $\mathrm{B}$, and we assume that the link characteristics are reciprocal, from $\mathrm{A}$ to $\mathrm{B}$ and $\mathrm{B}$ to $\mathrm{A}$. Two OFDM signals, one for each direction, occupy a total bandwidth equal to $B[\mathrm{~Hz}]$. As previously described, a portion of the sub-carriers operates in FD mode while the rest in HD. For transmitting in the FD portion of the spectrum, both devices are equipped with a SIC module needed for contrasting efficiently the selfinterference w.r.t. the signal of interest, which is received heavily attenuated by the propagation path loss and the random fading. In Fig. 1, a high-level block diagram of an OFDM FD transceiver with a SIC module is sketched.

The wireless propagation channel model between $\mathrm{A}$ and $B$, assumed reciprocal in the two directions, is modeled by a flat frequency path loss (PL) contribution and a random fading frequency selective transfer function $H_{C}(f)$. The analysis has been conducted for two channel models: (a) an AWGN channel model that introduces additive white Gaussian noise, assuming all over the bandwidth a normalized channel transfer function $H_{C}(f)=1$, and (b) a frequency selective fading channel model where the received signal on the $i$ th subcarrier with center frequency $f_{i}$ is affected by the channel transfer function $H_{C}\left(f_{i}\right)=H_{i}$. The frequency selective channel is simulated either by a Rayleigh channel model with different coherence bandwidths or by using the multipath intensity profile of the ITU-VehA channel model [26].

In case of Rayleigh fading model, in order to reproduce the general impact of a frequency selective channel according to the coherence bandwidth definition $B_{C}$, i.e., the range of frequencies over which the channel response is highly correlated, we consider a simplified channel, as proposed in [16] where:

- The bandwidth of the signal is divided into equal sub-bands of width $B_{C}[\mathrm{~Hz}]$, where the channel is assumed flat;

- In each sub-band, the complex channel amplitude is a zero-mean Gaussian random process, with Rayleigh distributed envelope, and the channel coefficients in different sub-bands are independent and identically distributed;

- As representative of the channel frequency selectivity, the single parameter $\lambda_{C}=B_{C} / B$ allows to reproduce the frequency selectivity effect of different channel models.

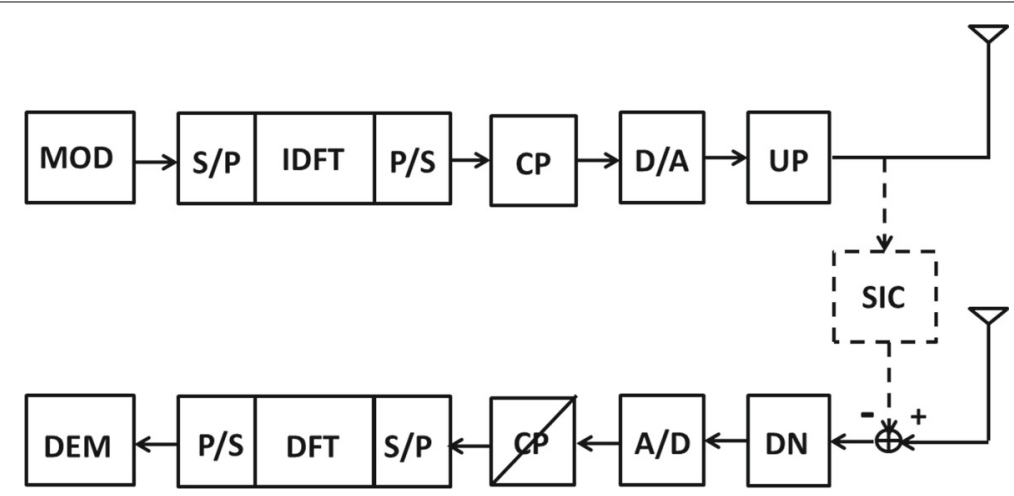

Fig. 1 FD transceiver. High-level block diagram of the FD transceiver with the SIC module 
Concerning the path loss (PL), we have adopted the path loss (PL) formula proposed in 3GPP Recommendation [26] for an Urban LoS (Line of Sight) environment:

$$
10 \cdot \log _{10}(\mathrm{PL}(d))=30.18+26 \cdot \log _{10}(d),
$$

where the link distance $d$ is in meters.

We summarize the assumptions of our system as:

- The channel is reciprocal in the two directions (i.e., A to $\mathrm{B}$ and $\mathrm{B}$ to $\mathrm{A}$ );

- The overall propagation channel transfer function is characterized by the path loss PL, dependent on the link distance $d$, and the frequency selective Rayleigh fading $H_{C}(f)$;

- The effect of transceiver non-idealities is modeled at the receiver as an error vector magnitude (EVM) noise with average power proportional to the received power $P_{R}$, as $P_{R} / \gamma_{E}$ [25];

- An echo cancelation circuit, with capability defined by the parameter SIC, is able to mitigate the selfinterference generated by bidirectional transmission in FD sub-carriers. The residual self-interference signal after the canceler is assumed flat with power $P_{T} / \mathrm{SIC}$, where $P_{T}$ is the transmit power on each sub-carrier. In practice, some frequency dependence could be present in the residual self-interference power, but we assume that its effect on the final signal-to-interference-plus-noise ratio can be included in the frequency selectivity of the channel;

- The effect of the leakage that the FD sub-carriers generate into the HD ones is modeled as an EVM noise with average power proportional to the residual self-interference generated by the FD sub-carriers $\mathrm{PDP} \cdot P_{T} / \mathrm{SIC}$, as PDP $\cdot\left(P_{T} / \mathrm{SIC}\right) / \gamma_{E}$.

According to these assumptions, the power of the received signal of interest $P_{R, i}$ on the $i$ th sub-carrier at frequency $f_{i}$, assuming all the sub-carriers at the same transmit power $P_{T}$ transmit and receive antenna gains $G_{T} \cdot G_{R}=G_{\text {ant }}$ and channel power gain $g_{i}=\left|H_{i}\right|^{2}$, is given by:

$$
P_{R, i}=\frac{P_{T} \cdot G_{\mathrm{ant}} \cdot g_{i}}{\mathrm{PL}}
$$

and the value of the signal-to-noise ratio on HD subcarriers $\gamma_{\mathrm{HD}, i}(2)$ is simply given by:

$$
\gamma_{\mathrm{HD}, i}=\frac{P_{R, i}}{P_{N}+P_{R, i} / \gamma_{E}+\operatorname{PDP} \cdot P_{T} /\left(\mathrm{SIC} \cdot \gamma_{E}\right)} .
$$

with the thermal noise power $P_{N}$, the EVM noise $P_{R, i} / \gamma_{E}$, and the leakage from FD sub-carriers PDP $\cdot P_{T} /\left(\mathrm{SIC} \cdot \gamma_{E}\right)$.

Analogously, considering that on FD sub-carriers the residual self-interference power is given by $P_{T} / \mathrm{SIC}$, the signal-to-interference-plus-noise ratio $\gamma_{\mathrm{FD}, i}$, in (3), is given by:

$$
\gamma_{\mathrm{FD}, i}=\frac{P_{R, i}}{\left(P_{T} / \mathrm{SIC}\right)+P_{N}+P_{R, i} / \gamma_{E}} .
$$

Notice that, in the presence of random fading, the notation includes the ' $j$ ' subscript for denoting the $j$ th fading realization, i.e.:

$$
\begin{aligned}
& \gamma_{\mathrm{HD}, i, j}=\frac{P_{R, i, j}}{P_{N}+P_{R, i, j} / \gamma_{E}+\mathrm{PDP} \cdot P_{T} /\left(\mathrm{SIC} \cdot \gamma_{E}\right)}, \\
& \gamma_{\mathrm{FD}, i, j}=\frac{P_{R, i, j}}{\left(P_{T} / \mathrm{SIC}\right)+P_{N}+P_{R, i, j} / \gamma_{E}} .
\end{aligned}
$$

\section{Methods for the analysis of rate gain regions}

The analysis in AWGN channel is a preliminary step for the Rayleigh frequency selective model, separated in Section 4.1. In AWGN, all the sub-carriers experience the same received power:

$$
P_{R, i}=P_{R}
$$

for $i=1,2, \cdots, N$, and consequently, they have the same $\gamma_{\mathrm{HD}, i}=\gamma_{\mathrm{HD}}$ and $\gamma_{\mathrm{FD}, i}=\gamma_{\mathrm{FD}}$ and the same $\eta_{\mathrm{HD}, i}=\eta_{\mathrm{HD}}$ and $\eta_{\mathrm{FD}, i}=\eta_{\mathrm{FD}}$ for $\mathrm{HD}$ and $\mathrm{FD}$ modes, respectively. In order to analyze the spectral efficiency gain region w.r.t. the value of PDP, we make explicit in (4) the dependence of the spectral efficiency expression at varying PDP as:

$$
\eta_{\mathrm{PD}}=\mathrm{PDP} \cdot \eta_{\mathrm{FD}}+(1-\mathrm{PDP}) \cdot \eta_{\mathrm{HD}},
$$

and we study the sign of its derivative w.r.t. PDP, for $0<$ PDP $<1$,

$$
\frac{\delta \eta}{\delta \mathrm{PDP}}=\eta_{\mathrm{FD}}-\eta_{\mathrm{HD}}
$$

It is straightforward to see two simple cases emerging:

$$
\begin{aligned}
& \eta_{\mathrm{FD}}>\eta_{\mathrm{HD}} \Longrightarrow \frac{\delta \eta}{\delta \mathrm{PDP}}>0 \Longrightarrow \mathrm{PDP}_{\mathrm{OPT}}=1, \\
& \eta_{\mathrm{FD}}<\eta_{\mathrm{HD}} \Longrightarrow \frac{\delta \eta}{\delta \mathrm{PDP}}<0 \Longrightarrow \mathrm{PDP}_{\mathrm{OPT}}=0,
\end{aligned}
$$

highlighting that the optimal design scheme coincides with a full FD scheme $\left(\mathrm{PDP}_{\mathrm{OPT}}=1\right)$ when $\eta_{\mathrm{FD}}>\eta_{\mathrm{HD}}$ and with a full $\mathrm{HD}$ transmission $\left(\mathrm{PDP}_{\mathrm{OPT}}=0\right)$ when $\eta_{\mathrm{FD}}<\eta_{\mathrm{HD}}$. The definition of the spectral efficiency gain region is: 


$$
\left(1+\gamma_{\mathrm{FD}}\right)^{2}>\left(1+\gamma_{\mathrm{HD}}\right)
$$

that turns out to be:

$$
\gamma_{\mathrm{FD}}^{2}+2 \gamma_{\mathrm{FD}}>\gamma_{\mathrm{HD}}
$$

Hence, from (12) and (13), for $g_{i}=1$, the spectral efficiency gain condition becomes $\mathrm{SIC}>\mathrm{SIC}_{0, \mathrm{AWGN}}$, with $\mathrm{SIC}_{0, \mathrm{AWGN}}$ computed as in (17).

$\operatorname{SIC}_{0, \mathrm{AWGN}}$
$=\frac{2 P_{T}\left(1-2 \frac{\mathrm{PDP}}{\gamma_{E}}\right)}{\left(\mathrm{PDP} / \gamma_{E}\right)\left(P_{R}\left(1+\frac{2}{\gamma_{E}}\right)+2 P_{N}\right)+\sqrt{\frac{\mathrm{PDP}}{\gamma_{E}^{2}}\left(P_{R}\left(1+\frac{2}{\gamma E}\right)+2 P_{N}\right)^{2}+4 P_{R}\left(P_{N}+\frac{P_{R}}{\gamma E}\right)\left(1-2 \frac{\mathrm{PDP}}{\gamma E}\right)}}$.

In particular, assuming in (17) $\gamma_{E}=\infty$ and $P_{N} \ll$ $\frac{P_{T} \cdot G_{\text {ant }}}{P L}$, i.e., $\gamma_{\mathrm{HD}, \mathrm{AWGN}} \gg 1, \mathrm{SIC}_{0, \mathrm{AWGN}}$ is reduced to:

$$
\mathrm{SIC}_{0, \mathrm{AWGN}}=\sqrt{\frac{P_{T} \cdot \mathrm{PL}}{P_{N} \cdot G_{\mathrm{ant}}}} .
$$

Some observations can be made looking at the formulae:

- For $\mathrm{SIC} \leq \mathrm{SIC}_{0, \mathrm{AWGN}}$, where $\eta_{\mathrm{HD}} \geq \eta_{\mathrm{FD}}$, PD schemes are not able to provide any efficiency gain, and they suffer from an efficiency loss, which increases as SIC decreases. In this case, the best choice is a pure HD system (PDP $=0$ ) with spectral efficiency $\eta_{\min }=\eta_{\mathrm{HD}}$ and efficiency gain $\eta_{G \text {, min }}=0$;

- For SIC $>\mathrm{SIC}_{0, \mathrm{AWGN}}$, the efficiency gain of the PD scheme increases linearly with PDP with slope $\eta_{\mathrm{FD}}-\eta_{\mathrm{HD}}$ as in (15), and the best design is a full FD system $(\mathrm{PDP}=1)$;

- For values of SIC well above the threshold, the residual self-interference becomes negligible w.r.t. the thermal noise, i.e., $\gamma_{\mathrm{FD}}=\gamma_{\mathrm{HD}}$, and the optimal design is with $\mathrm{PDP}=1$, providing the maximum performance $\eta_{\max }=2 \eta_{\mathrm{HD}}$ and $\eta_{G, \max }=100 \%$;

- For SIC values slightly above the threshold, i.e., when $\gamma_{\mathrm{FD}}$ is dominated by the self-interference, spectral efficiency and efficiency gain follow approximately a logarithmic function.

\subsection{Frequency selective Rayleigh channels}

In multi-carrier systems with frequency selective fading, the PD strategies for partitioning the spectrum in HD and FD portions can take advantage from the flexibility of the system. For a Rayleigh fading channel, the channel power gain $g_{i} \geq 0$ that appears in the received power on the $i$ th sub-carrier (11) is exponentially distributed with probability density function $f\left(g_{i}\right)$, cumulative density function $F\left(g_{i}\right)$, and mean value $\bar{g}_{i}=E\left[g_{i}\right]$ as:

$$
f\left(g_{i}\right)=e^{-g_{i}} ; F\left(g_{i}\right)=1-e^{-g_{i}} ; \bar{g}_{i}=1 .
$$

Differently from the frequency flat AWGN channel, in which the threshold $\mathrm{SIC}_{0, \mathrm{AWGN}}$ discriminates clearly the convenience between FD and HD transmissions, the presence of frequency selective fading requires a more elaborated response. Following the approach in (14)-(18), for each $i$ th sub-carrier, we define a $\mathrm{SIC}_{0, i}$ threshold obtained substituting $P_{R}$ with $P_{R, i}=\frac{P_{T} \cdot G_{\text {ant }} \cdot g_{i}}{P L}$ in (17).

Now, the performance analysis of the allocation strategies SPD and BPD requires a probabilistic approach, through the determination of the statistics of $\mathrm{SIC}_{0, i}$ thresholds. Therefore, for these two allocation strategies, we define two SIC thresholds, $\mathrm{SIC}_{0}$ and $\mathrm{SIC}_{99}$, according to the efficiency gain regions defined in (8) and (9), respectively:

- When $\mathrm{SIC}=\mathrm{SIC}_{0}$,

$$
E_{j}\left[\eta_{\mathrm{FD}, i, j}\right]=E_{j}\left[\eta_{\mathrm{HD}, i, j}\right], \forall i \in \Pi_{\mathrm{FD}} ;
$$

- When $\mathrm{SIC}=\mathrm{SIC}_{99}$,

$$
\operatorname{Prob}_{j}\left(\eta_{\mathrm{FD}, i, j}=\eta_{\mathrm{HD}, i, j}\right) \geq 0.99, \forall i \in \Pi_{\mathrm{FD}} .
$$

In the case of $\gamma_{E}=\infty$ and $P_{N} \ll \frac{P_{T} \cdot G_{\text {ant }}}{\mathrm{PL}}$ (as in (18), i.e., for dominant self-interference w.r.t. AWGN and neglecting the effect of transceiver non-idealities), $\mathrm{SIC}_{0, i}$ thresholds turn out to be inversely proportional to the corresponding amplitude channel gain $g_{i}$, i.e.:

$$
\mathrm{SIC}_{0, i}=\sqrt{\frac{P_{T} \cdot \mathrm{PL}}{P_{N} \cdot G_{\mathrm{ant}} \cdot g_{i}}}=\operatorname{SIC}_{0, \mathrm{AWGN}} \cdot \frac{1}{\sqrt{g_{i}}},
$$

and we are able to derive the theoretical expressions of $\mathrm{SIC}_{0}$ and $\mathrm{SIC}_{99}$ thresholds for the two allocation strategies from the distribution of the amplitude channel gain. This derivation constitutes, as it will be shown in the numerical results, a reference for the system potential gain.

\subsubsection{Selective partial-duplex}

In order to maximize the spectral efficiency in a PD scheme, as the thresholds $\mathrm{SIC}_{0, i}$ in (22) are inversely proportional to the channel power gains $g_{i}$, the optimal allocation assigns the FD portion to the sub-carriers with the highest gains. Therefore, ordering the power channel gains with decreasing values $\left[g_{1}^{\prime}, g_{2}^{\prime}, \cdots, g_{N}^{\prime}\right]$, the FD sub-carriers will be the ones that experience the highest $N_{\mathrm{FD}}$ gains $\left[g_{1}^{\prime}, g_{2}^{\prime}, \cdots, g_{N_{\mathrm{FD}}}^{\prime}\right]$ corresponding to the SIC thresholds $\left[\mathrm{SIC}_{0,1}^{\prime}, \mathrm{SIC}_{0,2}^{\prime}, \cdots, \mathrm{SIC}_{0, N_{\mathrm{FD}}}^{\prime}\right]$ in increasing order. Denoted $\rho$ the SIC level of the system, if less than 
$N_{\mathrm{FD}}$ sub-carriers have thresholds $\mathrm{SIC}_{0, i}>\rho$, the proposed allocation is the best choice; if more than $N_{\mathrm{FD}}$ sub-carriers have $\mathrm{SIC}_{0, i}>\rho$, the selection of the highest $N_{\mathrm{FD}}$ gains $\left[g_{1}^{\prime}, g_{2}^{\prime}, \cdots, g_{N_{\mathrm{FD}}}^{\prime}\right]$ is again the best assignment. In fact, from (22), the SIC value $\rho$ corresponds to a gain value $\tilde{g}$ and, consequently, to a signal-to-noise ratio $\tilde{\gamma}_{\mathrm{HD}}$ and a signal-to-noise-plus-interference ratio $\tilde{\gamma}_{\mathrm{FD}}$ that satisfy (16):

$$
\tilde{\gamma}_{\mathrm{FD}}^{2}+2 \tilde{\gamma}_{\mathrm{FD}}=\tilde{\gamma}_{\mathrm{HD}}
$$

Now, for a generic gain $g>\tilde{g}$, the FD capacity gain over HD is:

$$
\eta_{\mathrm{G}}=\left(1+\frac{g}{\tilde{g}} \tilde{\gamma}_{\mathrm{FD}}\right)^{2}-\left(1+\frac{g}{\tilde{g}} \tilde{\gamma}_{\mathrm{HD}}\right)
$$

and, from (23):

$$
\eta_{\mathrm{G}}=\frac{g}{\tilde{g}}\left(\frac{g}{\tilde{g}}-1\right) \tilde{\gamma}_{\mathrm{FD}}^{2}
$$

Equation (24) shows the increasing trend of the capacity gain with $g$, justifying the best selection of the $N_{\mathrm{FD}}$ subcarriers with highest gains.

The derivation of the thresholds $\mathrm{SIC}_{0}$ and $\mathrm{SIC}_{99}$ starts from the knowledge of the cumulative distribution function of the power gain $F\left(g_{i}\right)$ (19); given a vector of $N$ power gains in ascending order, the cumulative distribution function $F_{N-N_{\mathrm{FD}}+1}(g)$, i.e., the probability that the first $N_{\mathrm{HD}}=N-N_{\mathrm{FD}}$ power gains are less than a defined $g$ value and the remaining $N_{\mathrm{FD}}$ power gains are greater than $g$, is:

$$
\begin{aligned}
& F_{N-N_{\mathrm{FD}}+1}(g)= \\
& \sum_{K=N-N_{\mathrm{FD}}+1}^{N}\left(\begin{array}{l}
N \\
K
\end{array}\right) F^{K}(g)(1-F(g))^{N-K} .
\end{aligned}
$$

In order to derive the expression of $\mathrm{SIC}_{0}$, we rewrite the condition (20), including $\gamma_{\mathrm{FD}} \gg 1$ and $\gamma_{\mathrm{HD}} \gg 1$, as

$$
\begin{aligned}
& E_{j}\left[\eta_{\mathrm{FD}, i, j}\right]=E_{j}\left[2 \log \left(1+\gamma_{\mathrm{FD}}\right)\right] \simeq 2 E_{j}\left[\log \left(\gamma_{\mathrm{FD}}\right)\right]= \\
& 2 \log \left(\frac{P_{T} \cdot G_{\mathrm{ant}}}{\mathrm{PL} \cdot\left(P_{T} / \mathrm{SIC}+P_{N}\right)}\right)+2 E_{j}\left[\log \left(g_{i, j}\right)\right],
\end{aligned}
$$

and then

$$
E_{j}\left[\eta_{\mathrm{HD}, i, j}\right]=\log \left(\frac{P_{T} G_{\mathrm{ant}}}{\mathrm{PL} \cdot P_{N}}\right)+E_{j}\left[\log \left(g_{i, j}\right)\right],
$$

with deriving $f_{N-N_{\mathrm{FD}}+1}(g)$ from:

$$
E_{j}\left[\log \left(g_{i, j}\right)\right]=\int \log (g) \cdot f_{N-N_{\mathrm{FD}}+1}(g) d g .
$$

Hence, comparing (26) and (27), the threshold $\mathrm{SIC}_{0}$ can be expressed as the threshold $\mathrm{SIC}_{0, \mathrm{AWGN}}$ (18) multiplied by a margin $\Delta_{0, \mathrm{SPD}}$ as:

$$
\mathrm{SIC}_{0, \mathrm{SPD}}=\Delta_{0, \mathrm{SPD}} \cdot \mathrm{SIC}_{0, \mathrm{AWGN}},
$$

where the margin $\Delta_{0, S P D}$ is equal to:

$$
\Delta_{0, \mathrm{SPD}}=\frac{1}{\sqrt{e^{E_{j}\left[\log \left(g_{i, j}\right)\right.}}},
$$

and depends on the fading distribution and on the PDP value (i.e., $N$ and $N_{\mathrm{FD}}$ ) according to (28).

Now, in order to derive the expression of $\mathrm{SIC}_{99}$ (21), we consider the cumulative distribution of

$$
\Lambda=1 / g
$$

$F_{\Lambda}(\Lambda)=\operatorname{Prob}\left(\frac{1}{g}<\Lambda\right)=\operatorname{Prob}\left(g>\frac{1}{\Lambda}\right)=1-F\left(\frac{1}{\Lambda}\right)$,

that for the SPD scheme is:

$$
F_{\Lambda, \mathrm{SPD}}(\Lambda)=1-F_{N-N_{\mathrm{FD}}+1}\left(\frac{1}{\Lambda}\right) .
$$

From the definition of the threshold SIC $_{99, S P D}$, we define $\Lambda_{99, \mathrm{SPD}}$ as the value that satisfies the condition $\operatorname{Prob}(\Lambda<$ $\left.\Lambda_{99, \mathrm{SPD}}\right)=0.99$, i.e., $F_{\Lambda, \mathrm{SPD}}\left(\Lambda_{99, \mathrm{SPD}}\right)=0.99$, and we call $\Delta_{99, \mathrm{SPD}}$ its square root:

$$
\Delta_{99, \mathrm{SPD}}=\sqrt{\Lambda_{99, \mathrm{SPD}}}
$$

Hence, analogously to the final expression derived for $\Delta_{0, \mathrm{SPD}}(29)$, from (22), we can express also the threshold SIC 99, SPD as the threshold SIC $_{0, \mathrm{AWGN}}$ (18) multiplied by the margin $\Delta_{99, \mathrm{SPD}}$ :

$$
\mathrm{SIC}_{99, \mathrm{SPD}}=\Delta_{99, \mathrm{SPD}} \cdot \mathrm{SIC}_{0, \mathrm{AWGN}} .
$$

Again, analogously to the margin $\Delta_{0, S P D}$, also the margin $\Delta_{99, S P D}$ depends on the fading distribution and on the values of $N$ and $N_{\mathrm{FD}}$ as in (31).

Notice that (25) assumes that the $N$ power gains are independent, i.e., the frequency selectivity factor is $\lambda_{C} \leq$ $1 / N$. For higher values of $\lambda_{C}$, the derivations can be rewritten replacing, in the formulae, $N$ and $N_{\text {FD }}$ with $\widetilde{N}=\left\lceil 1 / \lambda_{C}\right\rceil$ and $\tilde{N}_{\mathrm{FD}}=\left\lceil N_{\mathrm{FD}} /\left(N \lambda_{C}\right)\right\rceil$ respectively, introducing the impact of the frequency selectivity in the thresholds. Obviously, we expect a loss in the accuracy of the analytical results depending on the error introduced in the above approximations.

We remark that both expressions of $\mathrm{SIC}_{0, \mathrm{SPD}}$ and SIC $_{99, S P D}$ in (29) and (33) are composed of two factors: $\mathrm{SIC}_{0, \mathrm{AWGN}}$, which depends on the radio link parameters (transmitted power $P_{T}$, antenna gains $G_{\text {ant }}$, propagation path loss PL and consequently link distance $d$, noise power $P_{N}$ ), and a margin, $\Delta_{0, \mathrm{SPD}}$ and $\Delta_{99, \mathrm{SPD}}$, respectively, which depends on the fast fading channel (distribution, frequency selectivity $\lambda_{C}$ ) and on the PDP parameter of the PD scheme. 


\subsubsection{Block partial-duplex}

In the block partial case, the gain vector is not ordered anymore, and the FD sub-carriers are contiguous in a single block at the center of the bandwidth.

The derivation of the analytical expression of the thresholds $\mathrm{SIC}_{0, \mathrm{BPD}}$ and $\mathrm{SIC}_{99, \mathrm{BPD}}$, as for the SPD strategy, requires the knowledge of the distributions of the channel gain $g_{i}$ for the sub-carriers operating in FD mode. Here, the probability that randomly chosen $N_{\mathrm{FD}}$ power gains are less than a defined $g$ value, without caring about the values that the other $N-N_{\mathrm{FD}}+1$ channel gains assume, is expressed simply by $F_{N_{\mathrm{FD}}}(g)$ as:

$$
F_{N_{\mathrm{FD}}}(g)=(1-F(g))^{N_{\mathrm{FD}}} .
$$

Then, following the same steps of the thresholds derivation for the SPD strategy, also the thresholds $\mathrm{SIC}_{0, \mathrm{BPD}}$ and $\mathrm{SIC}_{99, \mathrm{BPD}}$ turn out to be the product of the threshold $\mathrm{SIC}_{0, \mathrm{AWGN}}$ by a margin, $\Delta_{0, \mathrm{BPD}}$ and $\Delta_{99, \mathrm{BPD}}$, respectively, which returns the impact of the channel, of the frequency selectivity $\lambda_{C}$ and of the PDP parameter:

$$
\begin{aligned}
& \mathrm{SIC}_{0, \mathrm{BPD}}=\Delta_{0, \mathrm{BPD}} \cdot \mathrm{SIC}_{0, \mathrm{AWGN}}, \\
& \mathrm{SIC}_{99, \mathrm{BPD}}=\Delta_{99, \mathrm{BPD}} \cdot \mathrm{SIC}_{0, \mathrm{AWGN}} .
\end{aligned}
$$

As noted for SPD, when the $N_{\mathrm{FD}}$ power gains are not independent, i.e., for frequency selectivity factors $\lambda_{C}>$ $1 / N,(34)$ has to be rewritten replacing $N_{\mathrm{FD}}$ with $\widetilde{N}_{\mathrm{FD}}=$ $\left\lceil N_{\mathrm{FD}} /\left(N \lambda_{C}\right)\right\rceil$.

It can be noticed that, differently from the SPD case, only $N_{\mathrm{FD}}$, and not $N_{\mathrm{FD}}$ and $N$, appears in the cumulative distribution of the channel gains $g_{i}$ operating in FD mode, i.e., $F_{N_{\mathrm{FD}}}(g)(34)$, and, consequently, in the margin expressions.

\section{Numerical results}

In this section, we report the results of the numerical analysis and the simulations of the PD system performance, with the aim of highlighting design trade-offs and spectral efficiency gain regions for practical application in OFDM point-to-point links. The main parameters of the simulated system are listed in Table 1. In a fixed bandwidth $B=10 \mathrm{MHz}$ with $N=1024$ subcarriers, for different link distances $d$, different values of the system parameters PDP and for the two PD partition strategies (BPD and SPD), the requirements on the SIC capability have been derived. First, PD transmission is analyzed in the absence of fading, i.e., on a AWGN channel. Then, the numerical results are extended to a Rayleigh frequency selective fading channel model; for each value of the frequency selectivity parameter $\lambda_{C}=[0.001,0.008,0.016,0.031,0.062,0.125,0.25,0.5,1]$,
Table 1 System parameters

\begin{tabular}{lll}
\hline$P_{T}$ & Fixed transmit power per sub-carrier & $-20 \mathrm{dBm}$ \\
$P_{N}$ & Additive white Gaussian noise power & $-127 \mathrm{dBm}$ \\
$G_{T}, G_{R}$ & Transmit and receive antenna gain & $20 \mathrm{~dB}$ \\
$\mathrm{PL}(d)$ & Path loss, 30.18+26 $\log _{10}(d[\mathrm{~m}])$ & {$[70: 90] \mathrm{dB}$} \\
$\mathrm{SIC}$ & Signal interference cancelation & {$[50: 150] \mathrm{dB}$} \\
$\mathrm{N}$ & Number of sub-carriers & 1024 \\
$\mathrm{PDP}$ & Partial-duplex parameter & {$[0: 0.1: 1]$} \\
$\lambda_{C}$ & Frequency selectivity parameter & {$[0.001: 1]$} \\
$d$ & Link distance & {$[30: 200] \mathrm{m}$} \\
$B$ & System bandwidth & $10 \mathrm{MHz}$ \\
\hline
\end{tabular}

corresponding to coherence bandwidths $B_{C}$ equivalent to $[1,8,16,32,64,128,256,512,1024]$ sub-carriers, 1000 realizations of the transfer function $H_{C}(f)$ are generated. Finally, the simulations are repeated for the ITU-VehA channel model.

\subsection{AWGN channel}

Figure 2 shows the behavior of $\mathrm{SIC}_{0, \mathrm{AWGN}}$ as a function of the distance $d$ for two different values of $G_{\text {ant }}$, $G_{\text {ant }}=40 \mathrm{~dB}$ (a) and $G_{\text {ant }}=0 \mathrm{~dB}$ (b). Moreover, each sub-figure shows the curves for three different values of $\gamma_{E}=\left[\infty, 10^{5}, 10^{3}\right]$. First, we can notice that the gain of the antennas $G_{\text {ant }}$ has a significant impact. For $\gamma_{E}=\infty$, as expected from the deterministic expression (18), decreasing the antennas gain reduces the received power and, consequently, self-interference becomes dominant with corresponding higher SIC requirements. Moreover, the antennas gain affects also the EVM noise power $P_{R} / \gamma_{E}$, for $\gamma_{E}<\infty$. Therefore, the greater the value of $G_{\text {ant }}$, the higher the impact of EVM noise, i.e., the $\gamma_{E}$ parameter, on the $\mathrm{SIC}_{0, \mathrm{AWGN}}$ value. On the other hand, the leakage contribution from FD to HD sub-carriers, which is related to the PDP parameter, has no effect on the $\mathrm{SIC}_{0, \mathrm{AWGN}}$ values.

\subsection{Frequency selective channel}

Figure 3 reports PD performance in terms of $E\left[\eta_{\mathrm{G}}\right]$, the spectral efficiency gain averaged on all the sub-carriers and all the fading realizations. The results refer to a frequency selective Rayleigh channel model with frequency selectivity parameter $\lambda_{C}=1 / N, \gamma_{E}=\infty$, SPD strategy and link distance $d=200 \mathrm{~m}$. The curves show that the SIC value that guarantees an efficiency gain depends on PDP; this suggests that a spectral efficiency advantage might be tuned by implementing a partial form of the FD scheme. Same results are reported in Fig. 4 vs. PDP for different values of SIC and are compared with the AWGN case: while in AWGN the gain increases linearly with PDP 


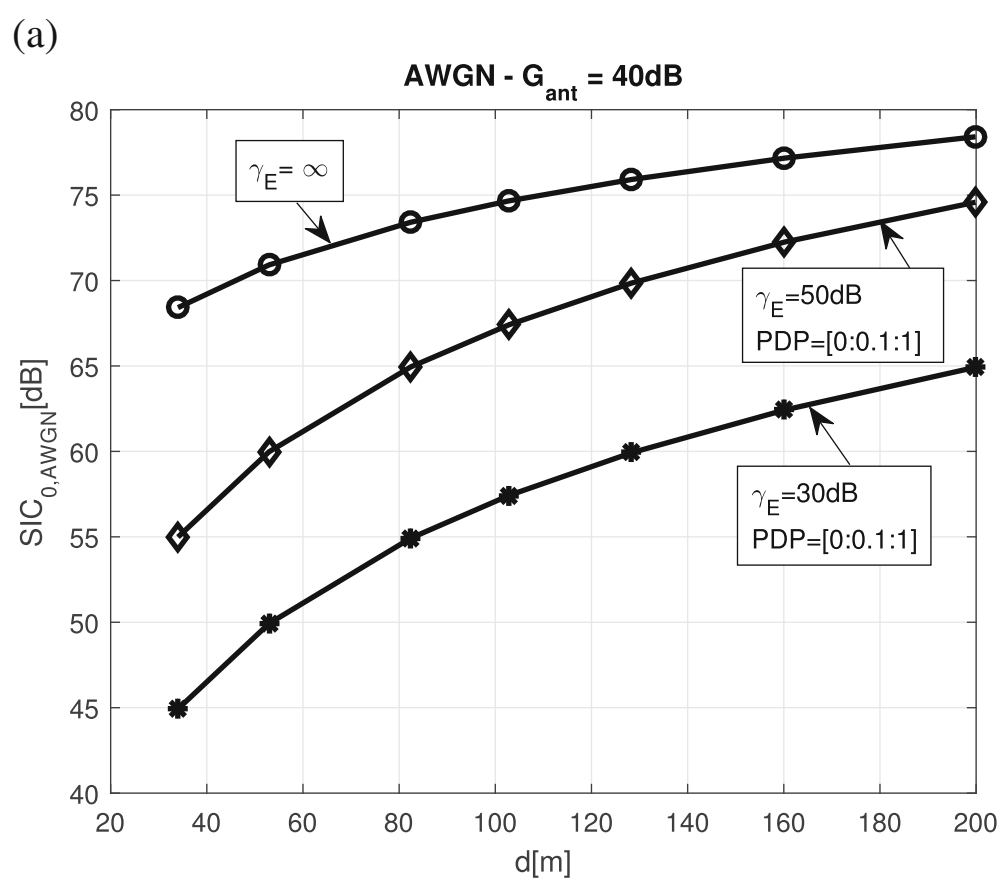

(b)

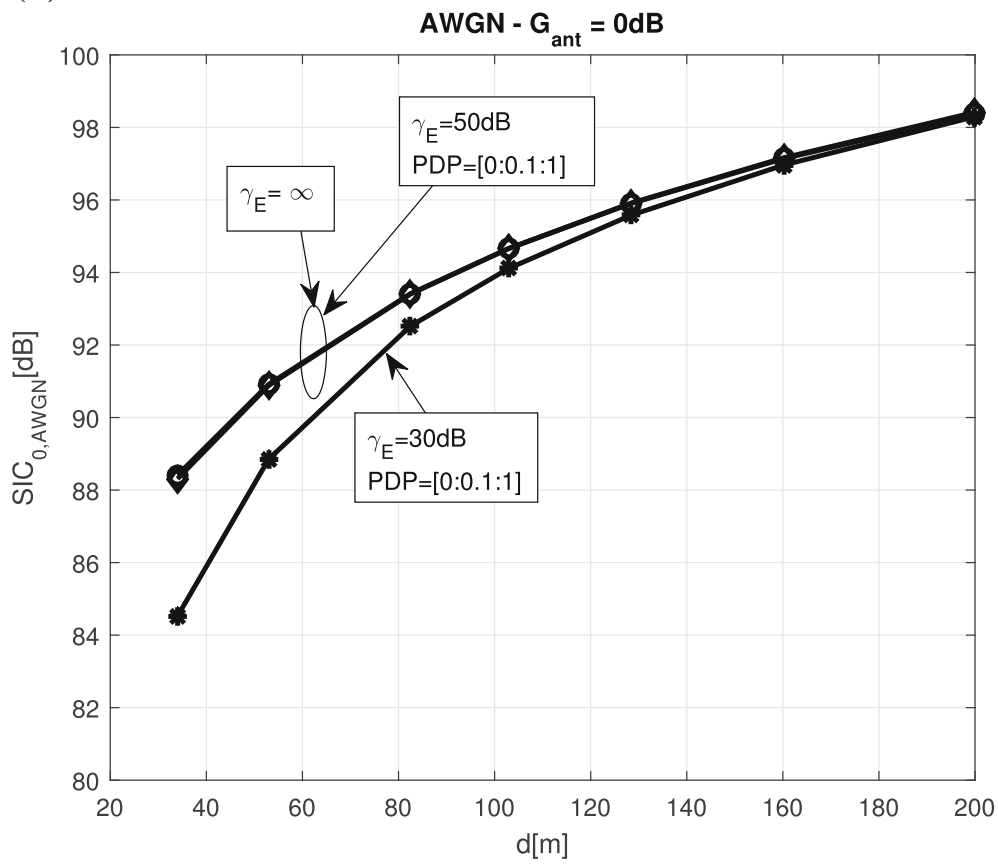

Fig. $2 \mathrm{SIC}$ threshold $\mathrm{SIC}_{0, \mathrm{AWGN}}$ for two different values of $G_{\text {ant }}$. Threshold $S \mathrm{C}_{0, \mathrm{AWGN}}\left[\mathrm{dB}\right.$ ] vs. distance $d$ for two different values of $G_{\text {ant }}, G_{\text {ant }}=40 \mathrm{~dB}$ (a) and $G_{a n t}=0 \mathrm{~dB}(\mathbf{b})$. Each sub-figure shows the curves for values of parameter $\gamma_{E}=\left[\infty, 10^{5}, 10^{3}\right]$ and PDP $=[0: 0.1: 1]$

for any value of SIC, in a channel with a high frequency selectivity, the SPD scheme can take advantage of a frequency diversity gain when PDP is low. In fact, the curve for $\mathrm{SIC}=80 \mathrm{~dB}$ shows a higher gain at $\mathrm{PDP}=0.5$ than at $\mathrm{PDP}=1$.
In order to better investigate the role of the parameters PDP and SIC on the spectral efficiency gain in PD schemes, we derived analytically and simulated the thresholds $\mathrm{SIC}_{0}, \mathrm{SIC}_{99}$ for the SPD and BPD strategies. Then, we plotted their margins w.r.t. $\mathrm{SIC}_{0, \mathrm{AWGN}}$, i.e., $\Delta_{0}$ 


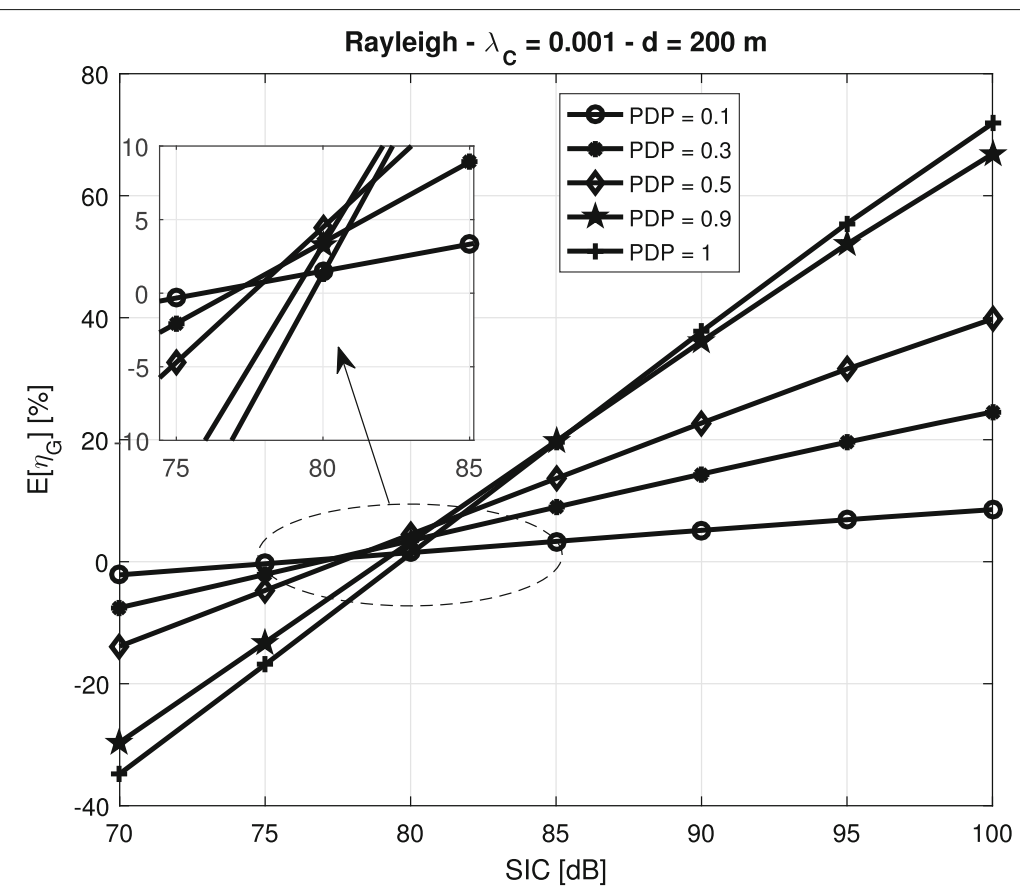

Fig. 3 PD spectral efficiency gain in frequency selective fading channel. Spectral efficiency gain $E\left[\eta_{\mathrm{G}}\right]$ [\%] vs. SIC at different values of PDP, for frequency selective Rayleigh channel model with $\lambda_{C}=0.001$, link distance $d=200 \mathrm{~m}$ and the SPD strategy

and $\Delta_{99}$, as defined in (29) and (33), and (35) and (36), respectively. Figures 5 and 6 show the resulting $\Delta_{0, S P D}$, $\Delta_{99, S P D}$ as a function of PDP for different values of the frequency selectivity factor $\lambda_{C}$, antenna gain $G_{\text {ant }}=40 \mathrm{~dB}$ and two different values of $\gamma_{E}: \gamma_{E}=\infty$ (a) and $\gamma_{E}=10^{3}$ (b). In the sub-figures (a), the analytical results (continuous lines) and the simulated ones (dotted lines) agree quite well and validate the theoretical analysis. The results in sub-figures (b), with the modeling of non-idealities and leakage noise, show increased values w.r.t. sub-figures (a)

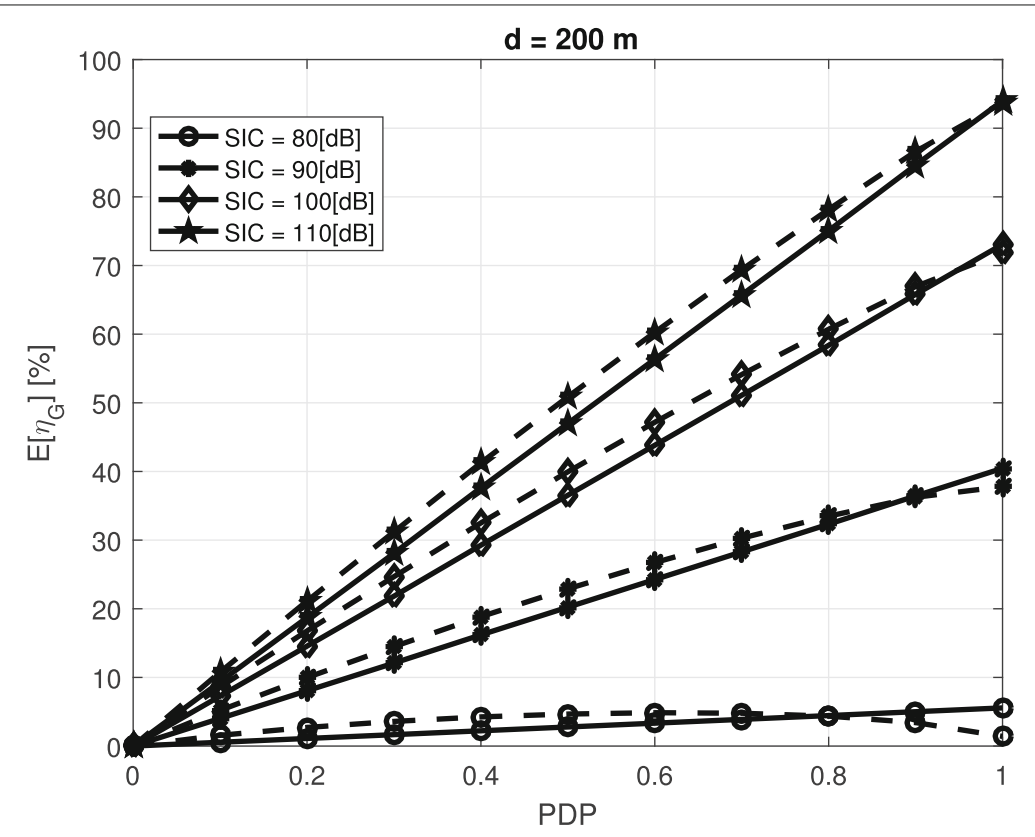

Fig. 4 PD spectral efficiency gain in AWGN and in frequency selective fading channel. Spectral efficiency gain $E\left[\eta_{\mathrm{G}}\right]$ [\%] vs. the parameter PDP for different values of SIC and link distance $d=200$ m; continuous lines refer to AWGN channel and dashed lines to Rayleigh channel with frequency selectivity $\lambda_{c}=0.001$ and the SPD strategy 
(a)

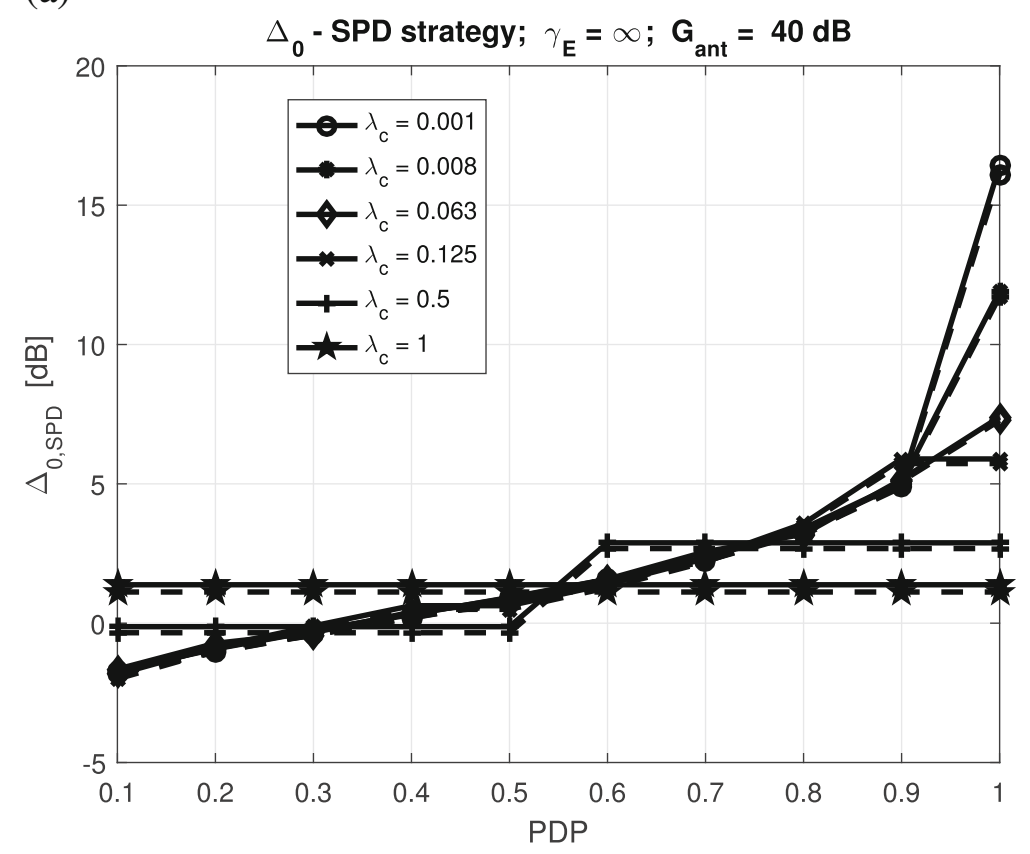

(b)

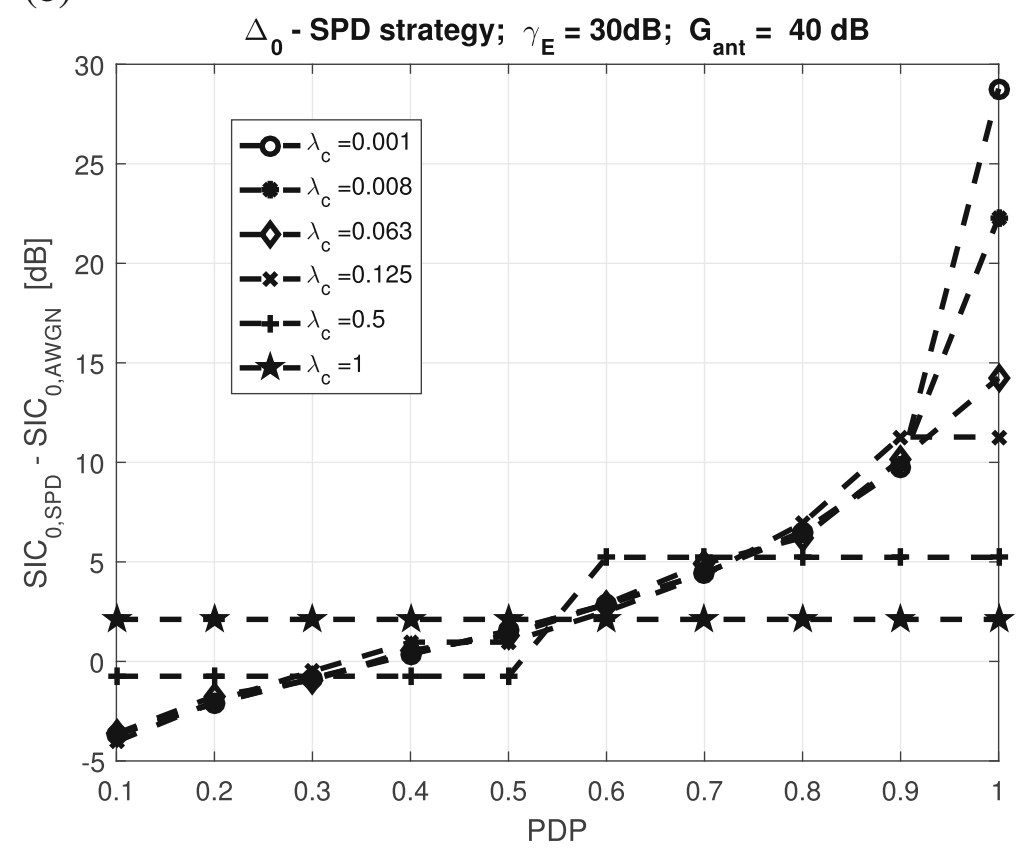

Fig. 5 Margin $\Delta_{0, S P D}$ for frequency selective Rayleigh channel model. a Margin $\Delta_{0, S P D}$ [dB] vs. the parameter PDP with $\gamma_{E}=\infty$ and $G_{a n t}=40 \mathrm{~dB}$,

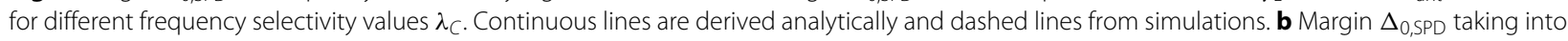
account non-idealities and leakage into the model $\left(\gamma_{E}=30 \mathrm{~dB}\right)$. The results are derived by simulating the Rayleigh fading realizations

but maintain a similar behavior w.r.t. PDP. The margins for the SPD strategy show variations from about $-2 \mathrm{~dB}$ to $17 \mathrm{~dB}$ with PDP for low values of $\lambda_{C}$. The selective strategy, exploiting the diversity gain offered by the frequency selectivity, for low PDP and low $\lambda_{C}$ shows negative margins. Interestingly, the curves show that, with a limited SIC capability, it may be an advantage to use PDP values lower than 1. 
(a)

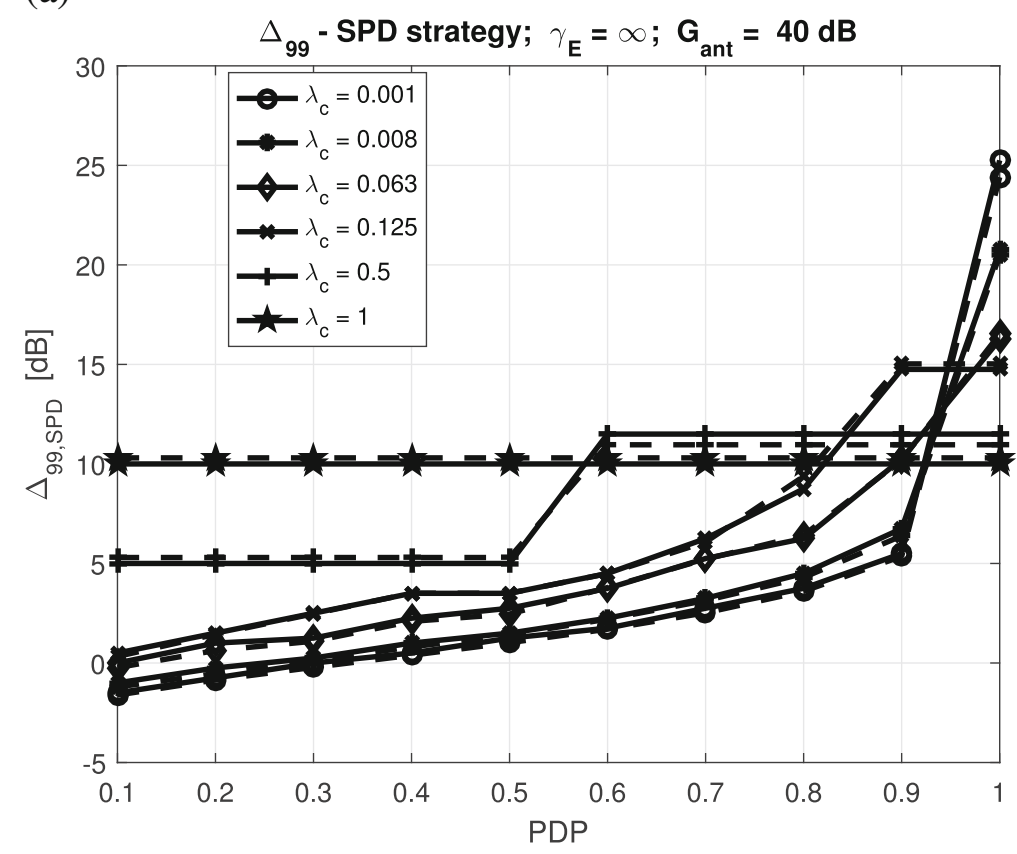

(b)

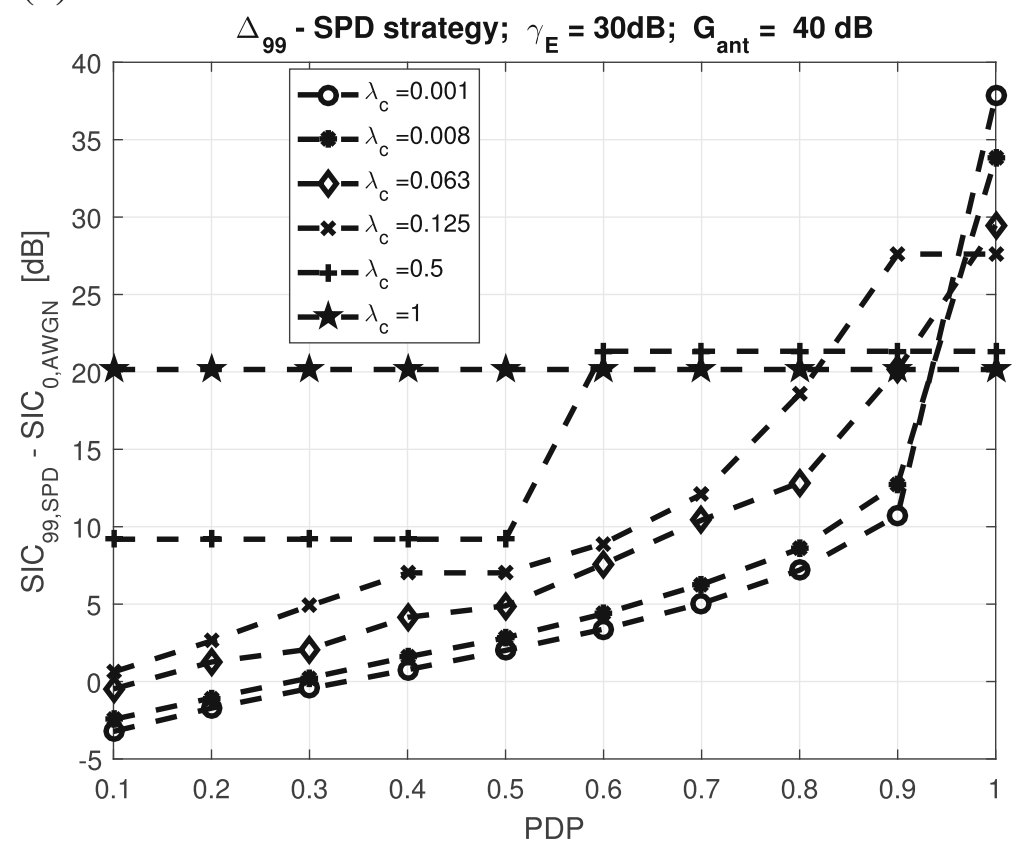

Fig. 6 Margin $\Delta_{99, S P D}$ for frequency selective Rayleigh channel model. a Margin $\Delta_{99, S P D}[\mathrm{~dB}]$ vs. the parameter PDP with $\gamma_{E}=\infty$ and $G_{a n t}=40 \mathrm{~dB}$, for different frequency selectivity values $\lambda_{C}$. Continuous lines are derived analytically and dashed lines from simulations. b Margin $\Delta_{99, S P D}$ taking into account non-idealities and leakage into the model $\left(\gamma_{E}=30 \mathrm{~dB}\right)$. The results are derived by simulating the Rayleigh fading realizations

Figures 7 and 8 refer to the BPD strategy; they report the margins $\Delta_{0, \mathrm{BPD}}$ and $\Delta_{99, \mathrm{BPD}}$ for $G_{\mathrm{ant}}=40 \mathrm{~dB}$ and $\gamma_{E}=\infty$. Also in this case, the simulation results (dotted lines) and the theoretical ones (continuous lines) agree except for some slight differences. We observe that the two strategies provide the same performance for the extreme cases PDP $=1$ and $\lambda_{C}=1$, as expected. But the margin for the BPD strategy is not 


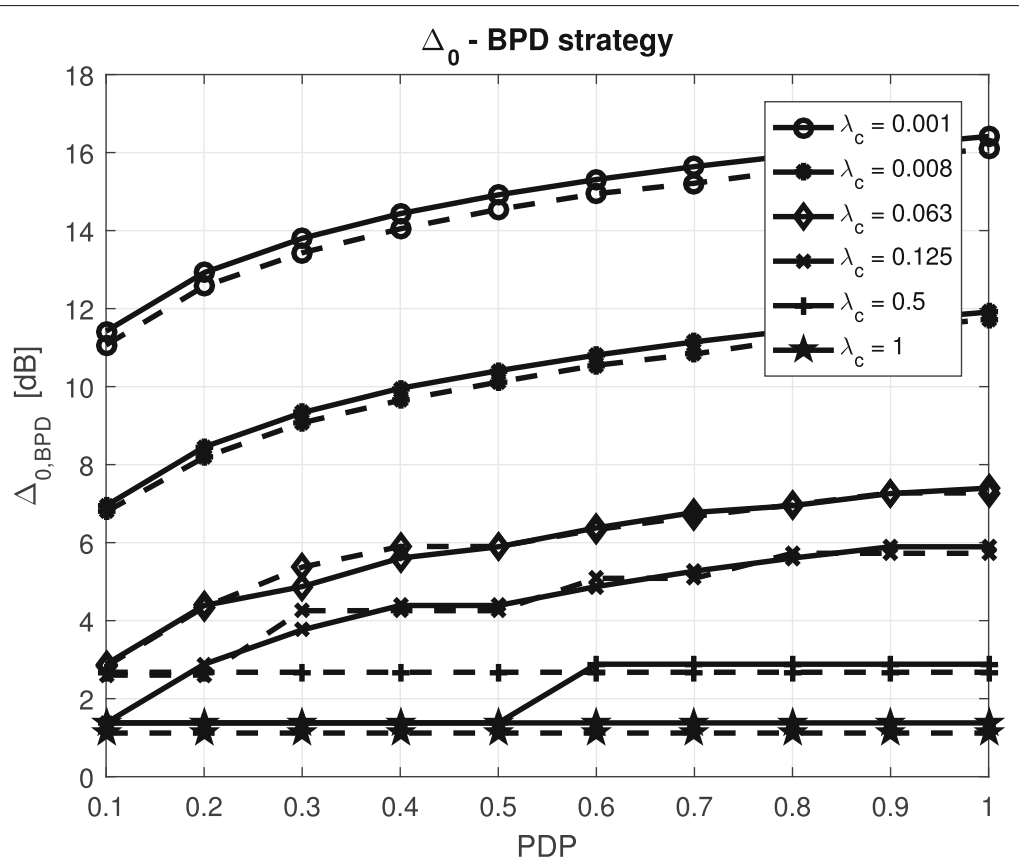

Fig. 7 Factor $\Delta_{0, B P D}$ for frequency selective Rayleigh channel model. Factor $\Delta_{0, B P D}$ [dB] vs. the parameter PDP for different frequency selectivity values $\lambda_{C}, \gamma_{E}=\infty$ and $G_{a n t}=40 \mathrm{~dB}$. Continuous lines are derived analytically and dashed lines from simulations

so sensitive to PDP variations, differently from the SPD strategy, as BPD is unable to select the best sub-carriers for FD. So, BPD shows the highest $\Delta_{99}$ at the lowest $\lambda_{C}$, as the probability to find heavily faded sub-carriers increases.

\subsection{ITU-VehA channel}

Figure 9 shows the ITU-VehA frequency autocorrelation vs. $\lambda_{C}$, here interpreted as $\Delta f / B$. As expected, the autocorrelation differs from our simplified model, which is reported on the same figure assuming $\lambda_{C}=0.5$. Then,

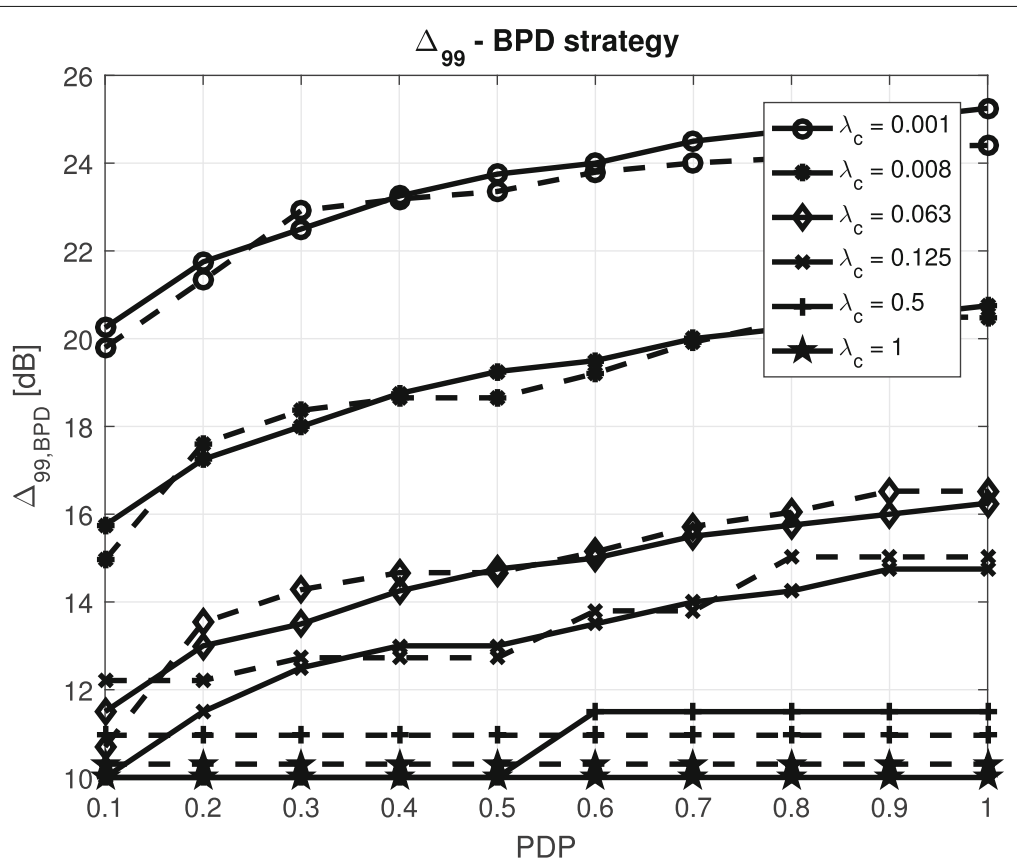

Fig. 8 Factor $\Delta_{99, B P D}$ for frequency selective Rayleigh channel model. Factor $\Delta_{99, B P D}[\mathrm{~dB}$ ] vs. the parameter PDP for different frequency selectivity values $\lambda_{C}, \gamma_{E}=\infty$ and $G_{a n t}=40 \mathrm{~dB}$. Continuous lines are derived analytically and dashed lines from simulations 


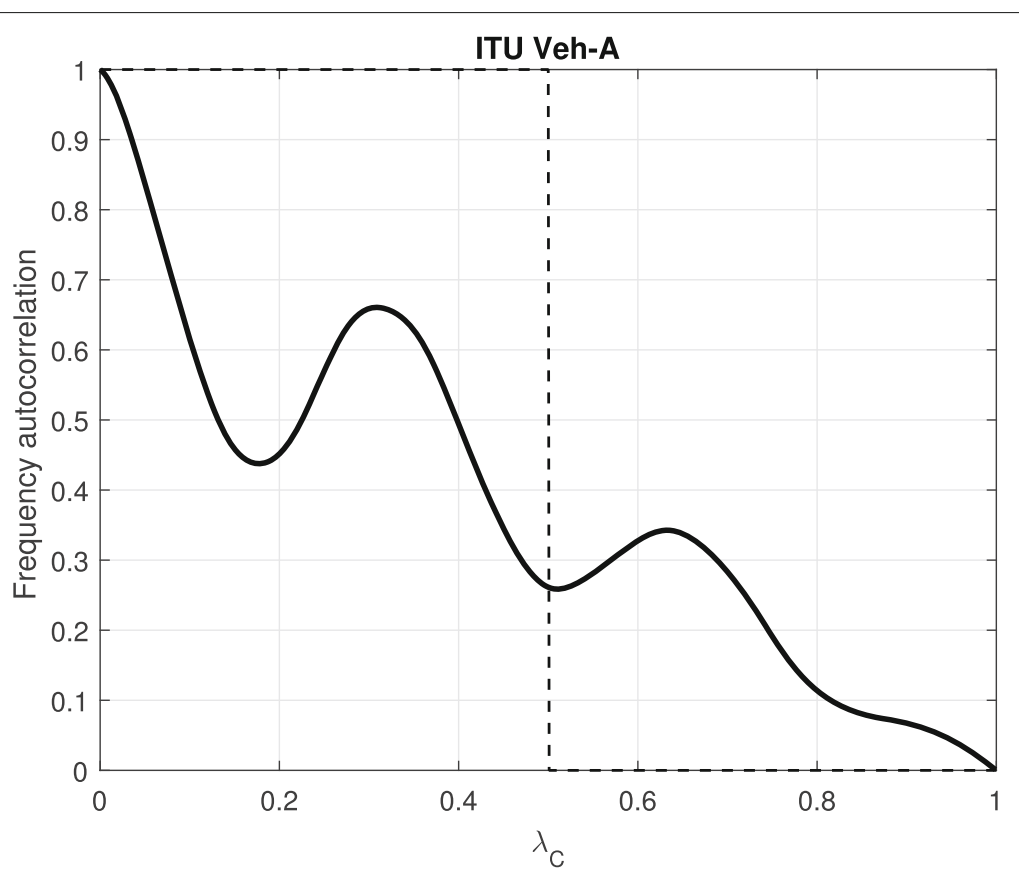

Fig. 9 Frequency autocorrelation of ITU-VehA channel model. Frequency autocorrelation of ITU-VehA channel model (continuous line) vs. the parameter $\lambda_{C}$, here defined as $\Delta f / B$. The ideal autocorrelation of the frequency selective model for $\lambda_{C}=0.5$ (dotted line) is reported for comparison

Fig. 10 reports the values of the four margins, $\Delta_{0}$ and $\Delta_{99}$ for both strategies, as a function of PDP. Due to the difference in the frequency correlation function of the two models, the correspondence between the two models looking at the $\Delta$ margin curves is not evident; anyway, ITU-VehA curves are inside the range of the $\lambda_{C}$ model. The curves for ITU-VehA model confirm the advantage of the SPD strategy w.r.t. BPD and the flexibility of the design of a PD scheme, in terms of trade-off between SIC capability and PDP. Particularly for the SPD strategy, $\Delta_{99}$ passes from $5 \mathrm{~dB}(\mathrm{PDP}=0.1)$ to $26 \mathrm{~dB}(\mathrm{PDP}=1)$, allowing to implement partial versions of the FD modality and providing spectral efficiency gains also with limited SIC capabilities.

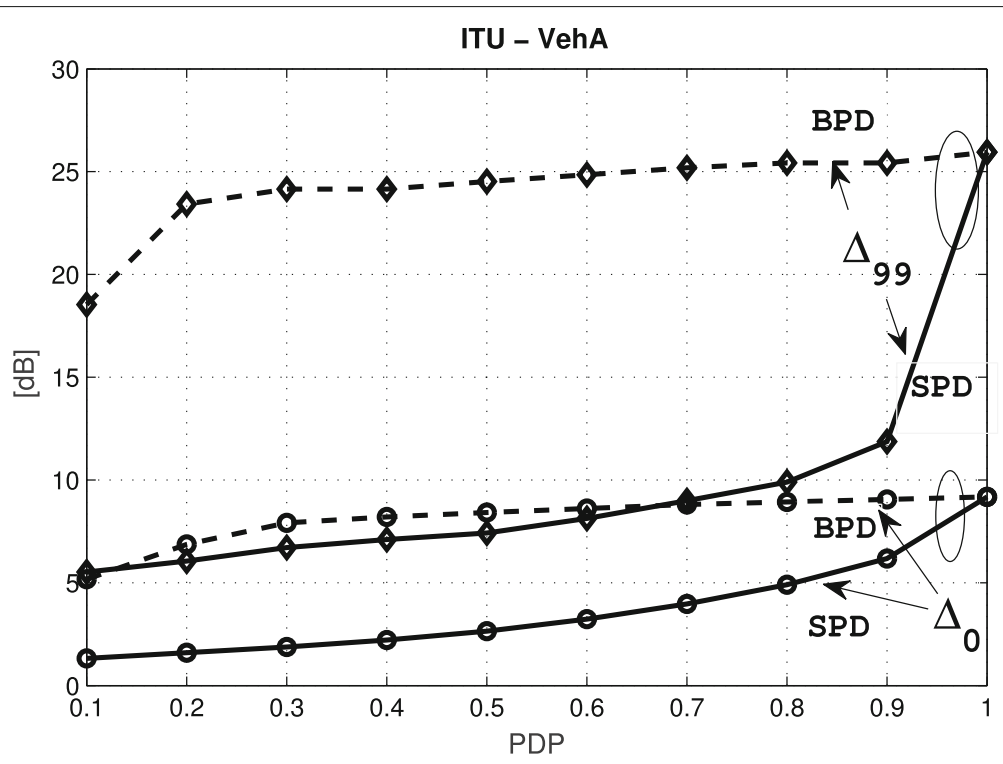

Fig. 10 Factors $\Delta_{0}$ and $\Delta_{9 g}$ for ITU-VehA channel model. Factors $\Delta_{0}$ and $\Delta_{9 g}$ Vs. PDP for ITU-VehA channel model. Continuous lines refer to the results obtained with the SPD strategy while dotted lines are obtained with the BDP strategy 


\section{Conclusions}

The self-interference canceler is a key component of wireless FD communication transceivers, limited in their application and performance by the extremely low levels of the received signal when compared to the residual self-interference signal. In this paper, we analyze the theoretical limits of a PD approach, characterized by FD operation in a portion of the available spectrum. The motivation behind this approach is the relaxation of the constraints on the self-interference canceler and the investigation of new trade-offs between the achievable spectral efficiency on the bidirectional link and the SIC capability. Starting from a general channel model characterized by Rayleigh frequency selective fading, we have investigated the role of the main system parameters from an analytical and simulation points of view, in order to highlight the potential of the PD solution.

\section{Abbreviations \\ AWGN: Additive white Gaussian noise; BPD: Block partial-duplex; EVM: Error vector magnitude; FD: Full-duplex; HD: Half-duplex; ITU-VehA: International Telecommunication Union - vehicular A; OFDM: Orthogonal frequency multiplexing division; PD: Partial-duplex; PDP: Partial-duplex parameter; SIC: Self-interference cancelation; SNR: Signal-to-noise ratio; SPD: Selective partial-duplex}

\section{Funding}

The authors certify that no funding has been received for the conduct of this study and the preparation of this manuscript.

\section{Authors' contributions}

All the authors have contributed jointly to the theoretical analysis and simulations, and they have approved the final manuscript.

\section{Competing interests}

The authors declare that they have no competing interests.

\section{Publisher's Note}

Springer Nature remains neutral with regard to jurisdictional claims in published maps and institutional affiliations.

\section{Author details}

${ }^{1}$ DEIB, Politecnico Di Milano, Milan, Italy. ${ }^{2}$ IEIIT, CNR, Milan, Italy.

Received: 20 March 2018 Accepted: 16 November 2018

Published online: 17 December 2018

\section{References}

1. S. Hong, J. Brand, J. I. Choi, M. Jain, J. Mehlman, S. Katti, P. Levis, Applications of self-interference cancellation in $5 \mathrm{G}$ and beyond. IEEE Commun. Mag. 52, 114-121 (2014)

2. M. Shikh-Bahaei, J. S. Choi, D. Hong, Full-duplex and cognitive radio networking for the emerging $5 \mathrm{G}$ systems. Wirel. Commun. Mob. Comput. Editorial (2018). https://doi.org/10.1155/2018/8752749

3. A. K. Khandani, Full-duplex (two-way) wireless: antenna design and signal processing (2012). Available: www.cst.uwaterloo.ca/reports/ antenna_design.pdf

4. D. Korpi, L. Anttila, M. Valkama, Nonlinear self-interference cancellation in MIMO full-duplex transceivers under crosstalk. EURASIP J. Wirel. Commun. Netw. 24 (2017). https://doi.org/10.1186/s13638-017-0808-4

5. M. Duarte, Full-duplex wireless: design, implementation and characterization. Ph.D. dissertation, Rice University (2012). https://core.ac. uk/download/pdf/10180083.pdf

6. E. Everett, M. Duarte, C. Dick, A. Sabharwal, in Conference Record of the 45th Asilomar Conference on Signals, Systems and Computers (ASILOMAR).
Empowering full-duplex wireless communication by exploiting directional diversity, (2011)

7. M. Jain, J. I. Choi, T. Kim, D. Bharadia, S. Seth, K. Srinivasan, P. Levis, S. Katti, $P$. Sinha, in Proceedings of the 17th annual international conference on Mobile computing and networking (ACM). Practical, real-time, full duplex wireless, (2011)

8. A. K. Khandani, in 13th IEEE Canadian Workshop in Information Theory (CWIT). Two-way (true full-duplex) wireless, (2013)

9. A. K. Khandani, Full-duplex wireless: design, implementation and characterization (2013). Available: http://www.cst.uwaterloo.ca/reports/ antenna_design.pdf

10. E. Aryafar, M. A. Khojastepour, K. Sundaresan, S. Rangarajan, M. Chiang, in Proceedings of the 18th annual international conference on Mobile computing and networking. Midu: enabling MIMO full duplex, (2012)

11. M. Duarte, A. Sabharwal, V. Aggarwal, R. Jana, K. Ramakrishnan, C. W. Rice, N. Shankaranarayanan, Design and characterization of a full-duplex multiantenna system for WiFi networks. IEEE Trans. Veh. Technol. 63(3), 1160-1177 (2014)

12. D. Bharadia, E. McMilin, S. Katti, Full duplex radios. ACM SIGCOMM Comput. Commun. Rev. 43(4), 375-386 (2013)

13. A. Sahai, G. Patel, A. Sabharwal, Pushing the limits of full-duplex: design and real-time implementation (2011). Available: http://arxiv.org/abs/ 1107.0607

14. M. Chung, M. S. Sim, J. Kim, D. K. Kim, C.-B. Chae, Prototyping real-time full duplex radios. IEEE Commun. Mag. 53, 56-63 (2015)

15. J. Bae, E. Park, K. Chang, H. Ju, Y. Han, in 82nd Vehicular Technology Conference IEEE (VTC Fall). In-band full-duplex system throughput analysis for Wi-Fi outdoor network, (2015)

16. L. Reggiani, L. Dossi, H. Barzegar, in 14th International Symposium on Wireless Communication Systems (VTC Spring), IEEE ComSoc. Extending the range of full-duplex radio with multi-carrier partial overlapping, (2017)

17. C. Yao, K. Yang, L. Song, Y. Li, in IEEE Conference Computer Communications Workshops (INFOCOM WKSHPS). X-duplex: adapting of full-duplex half-duplex, (2015)

18. J. Lee, T. Q. Quek, Hybrid full-/half-duplex system analysis in heterogeneous wireless networks. IEEE Trans. Wirel. Commun. 14(5), 2883-2895 (2015)

19. R. Keating, R. Ratasuk, A. Ghosh, in 83rd Vehicular Technology Conference (VTC Spring), IEEE. Performance analysis of full duplex in cellular systems, (2016)

20. Y. Shi, M. Ma, Two-way Relaying schemes in full duplex cellular system. EURASIP J. Wirel. Commun. Netw. 44 (2017). https://doi.org/10.1186/ s13638-017-0824-4

21. I. Randrianantenaina, H. Elsawy, H. Dahrouj, M.-S. Alouini, in IEEE International Conference on Communications (ICC). Interference management with partial uplink/downlink spectrum overlap, (2016)

22. A. AlAmmouri, H. ElSawy, O. Amin, M.-S. Alouini, In-band $\alpha$-duplex scheme for cellular networks: a stochastic geometry approach. IEEE Trans. Wirel. Commun. 15(10), 6797-6812 (2016)

23. A. AlAmmouri, H. ElSawy, M.-S. Alouini, Flexible design for $\alpha$-duplex communications in multi-tier cellular networks. IEEE Trans. Commun. 64(8), 3548-3562 (2016)

24. E. Ahmed, H. ElSawy, A.-M. Eltawil, A. Sabharwal, Rate gain region and design tradeoffs for full-duplex wireless communications. IEEE Trans. Wirel. Commun. 12(7), 3556-3565 (2013)

25. W. Li, J. Lilleberg, K. Rikkinen, On rate gain region analysis of half- and full-duplex OFDM communication links. IEEE J. Sel. Areas Commun. 32(9), 1688-1698 (2014)

26. 3GPP.TR.25.996, Project-Technical Specification Group Radio Access Network; Spatial channel model for multiple input multiple output (MIMO) simulations (Release 14). V14.0.0 (2017-03). (2017). Available: www.3gpp.org 